\title{
Upscaling of anisotropy in unsaturated Miller-similar porous media
}

\author{
Nadia Ursino \\ Soil Physics, Swiss Federal Institute of Technology Zurich, Schlieren
}

\author{
Kurt Roth \\ Institute of Environmental Physics, University of Heidelberg, Heidelberg, Germany \\ Thomas Gimmi and Hannes Flühler \\ Soil Physics, Swiss Federal Insțitute of Technology Zurich, Schlieren
}

\begin{abstract}
Geological and pedological processes rarely form isotropic media as is usually assumed in transport studies. Anisotropy at the Darcy or field scale may be detected directly by measuring flow parameters or may become indirectly evident from movement and shape of solute plumes. Anisotropic behavior of a soil at one scale may, in many cases, be related to the presence of lower-scale directional structures. Miller similitude with different pore-scale geometries of the basic element is used to model macroscopic flow and transport behavior. Analytical expressions for the anisotropic conductivity tensor are derived based on the dynamic law that governs the flow problem at the pore scale. The effects of anisotropy on transport parameters are estimated by numerical modeling.
\end{abstract}

\section{Introduction}

Processes such as sedimentation, compaction, frost action, and reorientation of the solid matrix are responsible for the creation of anisotropic natural porous media. Anisotropy can also be a characteristic of manufactured porous media like those made of irregularly shaped particles formed by extrusion or pelletting used in chemical engineering processes. Macroscopic effects of anisotropy are, for instance, the property of showing directionally varying conductivities or, referring to transport problems, dispersivities that may be larger or smaller relative to the corresponding isotropic medium, depending on the water content [McCord et al., 1990; Stephens and Heerman, 1988]. Flow through unsaturated porous media is often described based on saturated and relative conductivity functions. Many authors extended the concept of relative conductivity to anisotropic media by assuming that this property per se is directionally independent, but they used directionally differing saturated conductivities. Results predicted by such models do not agree with the observations where soils show different anisotropic, saturation-dependent behavior [McCord et al., 1990; Stephens and Heerman, 1988].

Anisotropic behavior is often attributed to the presence of layers or anisotropic correlation structures of variables describing flow and transport. Mualem [1985] evaluated the anisotropy factor of a soil consisting of many parallel layers. Green and Freyberg [1995], focusing on state-dependent anisotropy of conductivities caused by anisotropic correlation structures, compared their quasi-analytical upscaling with the stochastic approach of Yeh et al. [1985a, b]. The results of numerical upscaling in a three-dimensional anisotropic correlation structure were analyzed by Desbarats [1998]. All of the cited studies

Copyright 2000 by the American Geophysical Union.

Paper number 1999WR900320.

0043-1397/00/1999WR900320\$09.00 identified layering at the Darcy scale as the cause of macroscopic saturation-dependent anisotropy, leading to comparable results. Anisotropy at the pore scale, however, is rarely considered, except in some conceptual models assuming that the void system behaves as a bundle of capillary tubes [Bear et al., 1987] and in works based on percolation theory [Friedman and Seaton, 1996]. Upscaling of anisotropy of the pore structure yields a different macroscopic behavior when compared to upscaling anisotropy of the correlation structure.

The present study complements the findings of Bear et al. [1987] using fixed microscale principal directions for capillary flow. We will consider three different scales: the pore scale, the scale of the representative elementary volume (REV), as defined by Hubbert [1956] and Bear [1972], and the field scale. At the pore scale we consider the porous medium as an ensemble of pores embedded between particles. We introduce three anisotropic configurations of the void space: one in which only the density of the pores in the flow cross sections is anisotropic, one in which only the distribution of their diameters is anisotropic, and another in which density and pore size distribution are both anisotropic. Then, we derive the anisotropy factor of the conductivity tensor. Upscaling properties of the pore scale over a basic volume define the properties of the REV. The structure of the soil at the field scale is defined by a heterogeneous domain consisting of many REVs. We assume that the different basic elements of the heterogeneous medium are geometrically similar according to Miller and Miller [1956]. Heterogeneity then is the result of a random distribution of the Miller scaling factors. It is assumed that the correlation structure of the Miller scaling factors is isotropic. We evaluate transverse and longitudinal dispersivity by numerical simulation. Finally, we show the effect of anisotropy on transport by evaluating the ratio between the dispersivity of an anisotropic medium and the dispersivity of the corresponding isotropic medium for different water fluxes. 


\section{Theory}

2.1. Upscaling Procedures for the Anisotropic Conductivity Tensor

The REV is an operational definition which implies that the physical properties of the porous media at a scale smaller than that of the REV affect macroscale processes only in a statistical sense. As a consequence, any continuum model, being based on the characterization of the REV, relies on the assumptions regarding the subscale soil structure and the dynamics of fluids at the pore level. It is obvious that size, shape, and arrangement of solid particles, varying from soil to soil, have a profound impact on flow and transport. In this section we discuss the applicability of the scaling procedure developed by Miller and Miller [1956] to anisotropic media. We then present three different idealized anisotropic structures, and we derive the macroscopic anisotropy factor of the corresponding REVs.

Miller similitude is a physically based algorithm for defining scale-invariant capillary processes in unsaturated geometrically similar porous media [Miller and Miller, 1956]. It is based on the physical relationship between matric potential and pore size. Each REV is geometrically similar to the others and is identified by a characteristic length, that is, the size of grains and pores with respect to some reference element. Scaled relationships are derived assuming that the microscopic behavior of the liquid phase is governed by surface tension and the law of viscous flow. Isotropy of the macroscopic properties of the medium is defined by Miller and Miller [1956] as "a nonessential simplifying assumption." However, it is invoked to justify the assumption of parallelism between driving force and velocity in deriving Darcy's law from the viscous flow equation. We postulate that it is possible to choose the REV scale and the microscopic anisotropy factor in a way that the parallelism between driving force and velocity still holds when a gradient in one or the other of the main directions is imposed.

A crucial assertion of the Miller-scaling procedure is that, given a certain water content, similar elements of the porous medium are in a similar state. The concept of similar state requires postulating invariance of volumetric water content (classical Miller scaling) or invariance of areal pore size distribution (generalized scaling) [Sposito and Jury, 1990]. In the following the pore space distribution is the invariant quantity. Conceptually, the scaling factor applies to a complex system of stream tubes. In the anisotropic case this system has directiondependent average numbers of tubes and/or diameter sizes. We will analyze three different anisotropic geometrically similar microstructures. For each microstructure, at the pore scale, the anisotropy is quantified with a microscopic anisotropy factor $a_{i}$, with $i=1,2$, and 3 , that represents the ratio between the characteristic length of some geometric variable in the two main directions. At the larger scale the effect of the pore scale anisotropy $a_{i}$ is quantified by the ratio between the conductivities in the main directions (macroscopic anisotropy factors $A_{\imath}$ ). Figure 1 shows schematically the reference configuration for the isotropic (Figure 1a) and the three different anisotropic Miller-similar media that we analyze.

The first of the proposed models (Figure 1b) represents a medium with direction-dependent average number of pores. The microscopic anisotropy factor $a_{1}$ is defined as the ratio between the characteristic lengths of the grains in the two main directions. The oblate grains are all oriented in the same direction. The structure of a square REV is characterized by a larger number of pores in the direction of the smaller grain

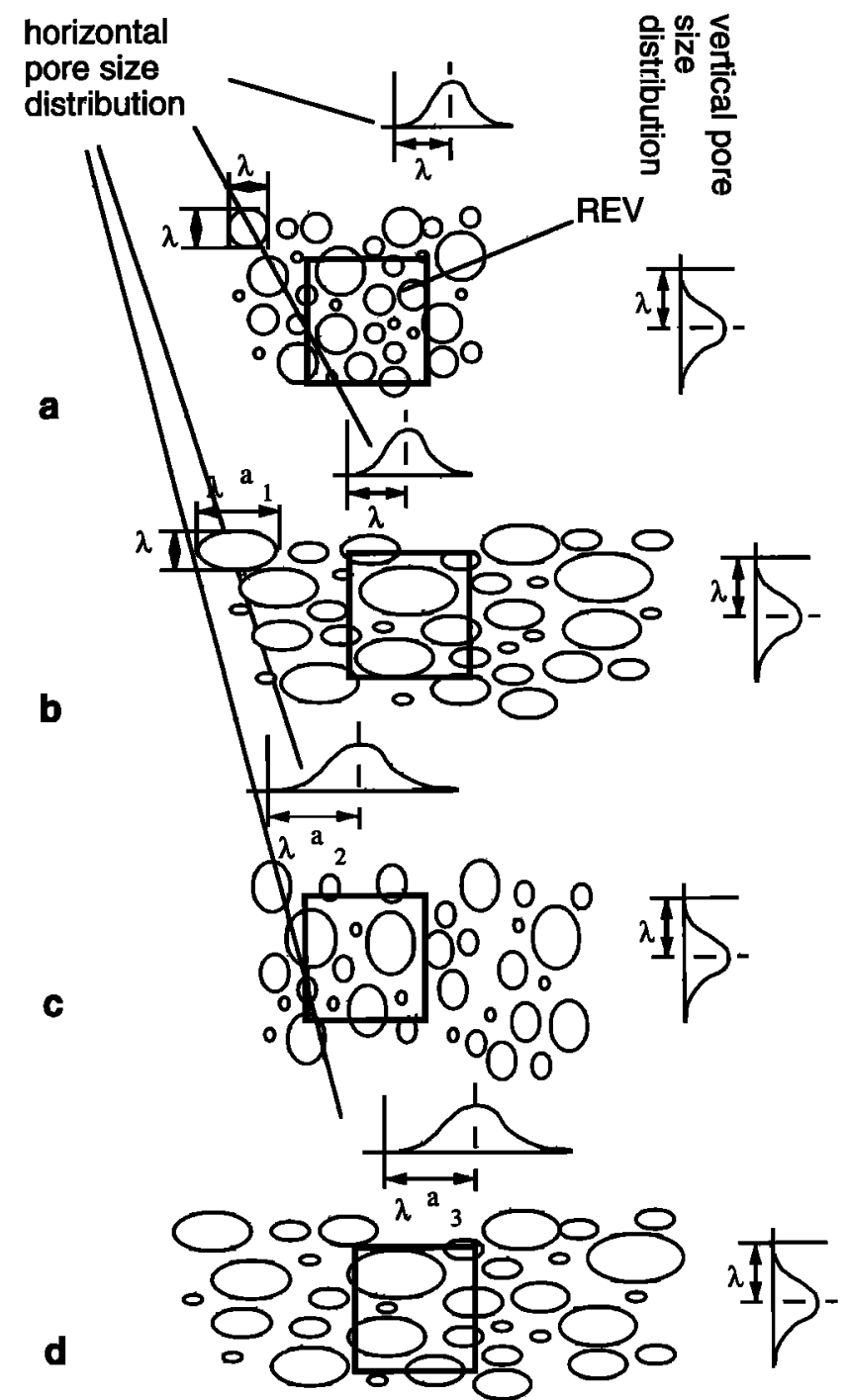

Figure 1. Schematic representation of basic porous structures of different Miller-similar media. (a) Isotropic, (b) anisotropic average number of pores (anp) and isotropic pore size distribution (psd), (c) anisotropic psd and isotropic anp, and (d) anisotropic anp and psd.

length and by a direction-independent statistical distribution of the pore diameters, coincident with that of the corresponding isotropic medium. The ratio between the average number of pores in the two main directions assumes the constant value $1 / a_{1}$. The effect of a direction-dependent pore density on the conductivity $K$ can be derived with the one-dimensional form of Darcy's law:

$$
K=\frac{q \Delta z}{\Delta \Psi}
$$

where $q[L / T]$ is the flux through all the conductive channels in the squared REV with dimension $\Delta z[L]$ and $\Delta \Psi[L]$ is the imposed pressure difference between the inlet and the outlet boundaries. Because the statistical pore size distribution is direction-independent, $q$ is proportional to the average number of pores contributing to the flow process at a given saturation degree. As a consequence, in this case, the macroscopic anisotropy factor $A_{1}$, defined as the ratio between the conduc- 
tivities of the REV in the two main directions, is equal to the inverse of the microscopic anisotropy factor $a_{1}$ and does not depend on saturation;

$$
A_{1}=K_{z} / K_{x}=1 / a_{1}
$$

where $K_{z}$ and $K_{x}$ are the vertical and horizontal components of the conductivity tensor, respectively.

The second model of anisotropy, shown in Figure 1c, describes direction-dependent distributions of pore sizes. The average number of pores is the same in the two main directions. To estimate the effect of the microscopic anisotropy factor $a_{2}$ on the macroscopic anisotropy factor $A_{2}$, we make use of the scaling theory of Miller and Miller [1956] twice, once when the mean gradient and flow are parallel to one of the main directions and once when they are parallel to the other, to obtain the scaled components of the conductivity tensor $K_{z}$ and $K_{x}$.

The functional relationships between soil properties at different locations of an isotropic heterogeneous domain which obeys Miller-Miller similarity are [Miller and Miller, 1956]:

$$
\begin{gathered}
\lambda^{\prime} \Psi^{\prime}(\Theta)=\lambda \Psi(\Theta) \\
\frac{K^{\prime}(\Theta)}{\lambda^{\prime 2}}=\frac{K(\Theta)}{\lambda^{2}},
\end{gathered}
$$

where $\Theta=\theta / \theta_{s}$ is the relative water saturation (ratio between the volumetric water content $\theta$ and the volumetric water content at saturation $\theta_{s}$ ), $K^{\prime}(\Theta)$ represents the dependence of the hydraulic conductivity on the relative water saturation in the neighborhood of a region with characteristic scale $\lambda^{\prime}$, and $K(\Theta)$ represents the same relationship in the neighborhood of a region with characteristic scale $\lambda$. We can also express the conductivities $K^{\prime}\left[\Psi^{\prime}(\Theta)\right]=K^{\prime}\left[\Psi(\Theta) \lambda / \lambda^{\prime}\right]$ and $K[\Psi(\Theta)]$ as a function of the corresponding pressures by eliminating $\Theta$. Equations (3) and (4) were used to derive the horizontal and vertical conductivities of an anisotropic element with respect to a reference conductivity. Assuming that $a_{2}$ is the ratio between the characteristic pore-scale lengths in the two main directions, we consider gradient and flow parallel to each one of them. Applying the Miller-scaling procedure for the cases in which the mean gradient is directed in each one of the two main directions, assuming that the average number of pores is the same, and expressing the conductivities as a function of pressure, we obtain

$$
\begin{gathered}
K_{z}(\Psi)=K(\Psi) \\
K_{x}(\Psi)=\frac{1}{a_{2}^{2}} K\left(\frac{\Psi}{a_{2}}\right) .
\end{gathered}
$$

$K_{z}$ and $K_{x}$ are the main components of the conductivity tensor (vertical and horizontal, respectively, with respect to Figure 1), and $K$ is the reference conductivity. For such an anisotropic medium the anisotropy factor $A_{2}$ is a function of capillary pressure head:

$$
A_{2}(\Psi)=\frac{K_{z}}{K_{x}}=\frac{a_{2}^{2} K(\Psi)}{K\left(\Psi / a_{2}\right)} .
$$

The third model proposed (Figure 1d) is a particular case of direction-dependent number of pores and pore sizes. Consider an isotropic reference state and stretch or squeeze it in one direction by a factor $a_{3}$. The ratio between the scaling factors of the pore sizes in the two main directions is $a_{3}$, and the ratio between average number of pores is $1 / a_{3}$. The two effects tend to compensate each other partially. The resulting conductivity anisotropy factor has the following form:

$$
A_{3}(\Psi)=\frac{K_{z}}{K_{x}}=\frac{a_{3} K(\Psi)}{K\left(\Psi / a_{3}\right)}
$$

\subsection{Numerical Solution of Flow and Transport Problems} in Anisotropic Heterogeneous Porous Media

Many numerical studies demonstrate the influence of smallscale heterogeneity on flow and transport through macroscopically isotropic Miller-similar media [Russo, 1991; Tseng and Jury, 1994; Roth, 1995]. We will now explore the effect of relaxing the hypothesis of isotropy on the basis of numerical simulations. We solve numerically the problem of flow and transport for anisotropic media with properties corresponding to models in Figures $1 b$ and 1c, as derived in section 2.1. The details of the implemented numerical procedure to determine longitudinal and transverse dispersivity are given by Roth [1995] and Roth and Hammel [1996]. We focus on a single realization of a random field of lognormally distributed scaling factors. The correlation structure of the logarithm of the scaling factor was characterized by a variance of $\sigma_{\lambda}^{2}=0.25$, by an isotropic correlation length of $L_{\lambda}=0.1 \mathrm{~m}$, and by an exponential autocovariance. We considered a two-dimensional domain extending $5 \mathrm{~m}$ in the horizontal direction and $6.25 \mathrm{~m}$ in the vertical direction, which is discretized with a grid constant of $0.025 \mathrm{~m}$. Uniform and constant water flow was assumed at the top boundary, and a pressure of zero was assumed at the bottom boundary. Different flow rates were used to examine the effect of differing average pressures. The soil properties were parameterized according to the Mualem-van Genuchten model [van Genuchten, 1980], which is used in the following form

$$
\begin{gathered}
\Psi(\Theta)=-\alpha^{-1}\left[\Theta^{n /(1-n)}-1\right]^{(1 / n)} \\
K(\Theta)=K_{s} \Theta^{1 / 2}\left\{1-\left[1-\Theta^{n /(n-1)}\right]^{1-1 / n}\right\}^{2} \\
\Theta=\theta / \theta_{s} .
\end{gathered}
$$

The vertical component of the conductivity was always kept constant $K_{z}=K(\Theta)$, whereas the horizontal component $K_{x}$ was varied according to the proposed relations (2) and (7). This provided, for a given water flux, an average water content independent of the microscopic anisotropy factors. The following parameters were used: $K_{s}=10^{-5} \mathrm{~m} \mathrm{~s}^{-1}, \alpha=4 \mathrm{~m}^{-1}, \theta_{s}=$ 0.4 , and $n=2$. These values are typical for a sand, which is a medium that might possibly follow Miller scaling. The flow problem was solved for steady state conditions. Afterward, the solute transport was explored by particle tracking. Particles are initially located at the upper boundary and are uniformly distributed horizontally with the exception of a $0.5 \mathrm{~m}$ neighborhood of the vertical boundaries to prevent boundary effects. We deal exactly with the problem setup defined by Roth [1995] and Roth and Hammel [1996] but with a new realization of scaling factors $\lambda$ and with anisotropic conductivities. Subscale dispersion (local dispersion) is neglected. The longitudinal dispersivity $\alpha_{l}$ and the transverse dispersivity $\alpha_{t}$ were estimated according to the following expressions:

$$
\begin{aligned}
\alpha_{l} & =\frac{z \operatorname{Var}[t(z)]}{2\langle t(z)\rangle^{2}} \\
\alpha_{t} & =\frac{\operatorname{Var}[\Delta x(z)]}{2 z},
\end{aligned}
$$



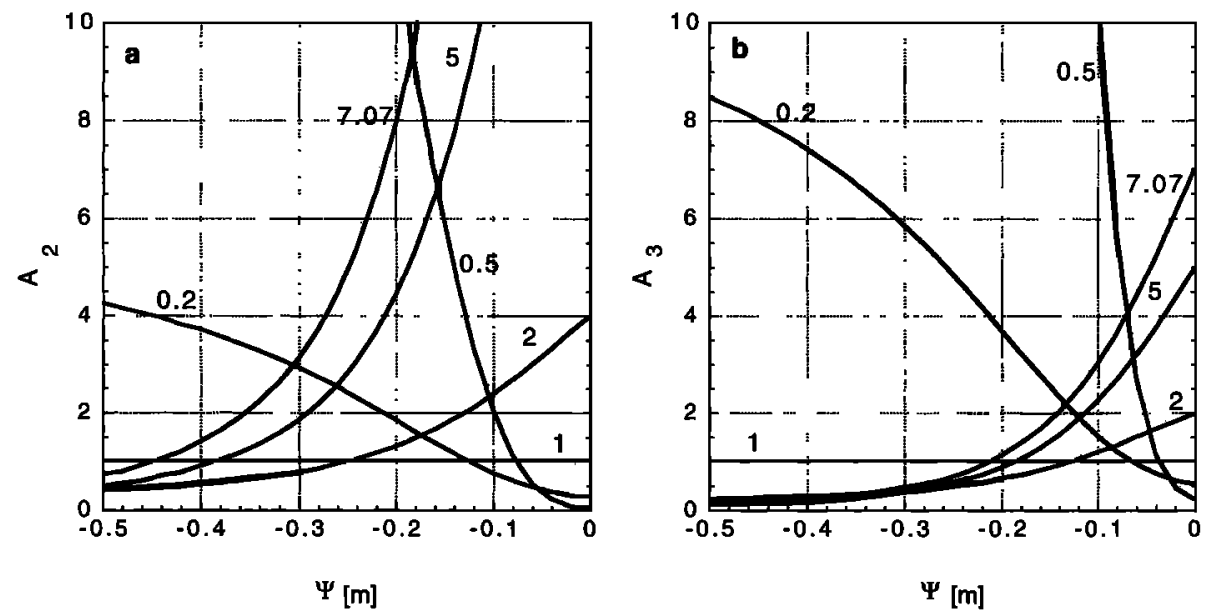

Figure 2. Pressure-dependent anisotropy factors $A_{2,3}=K_{z} / K_{x}$ for $a_{2,3}=0.2,0.5,1,2,5$, and 7.07. (a) Cases of anisotropic pore size distribution but isotropic number of pore $\left(A_{2}\right)$. (b) Cases of anisotropic number of pores and pore size distribution $\left(A_{3}\right)$. The horizontal line applies to the isotropic medium characterized by $a_{2,3}=1$. An intersection of another curve with the isotropic case indicates the switching point of the anisotropic medium where it behaves like isotropic.

where $t(z)$ is the arrival time at a certain depth $z$ of a generic particle, $\langle t(z)\rangle$ is its expectance, $\operatorname{Var}[t(z)]$ is its variance, and $\operatorname{Var}[\Delta x(z)]$ is the variance of the horizontal deviation of the generic particle with respect to the initial point of injection.

On the basis of the obtained numerical results, we estimated two anisotropy factors: the longitudinal and the transverse dispersivity ratios. The first is the ratio between the longitudinal dispersivity (LDR) derived for an anisotropic case and the one obtained assuming $a_{i}=1$ (isotropic case):

$$
\operatorname{LDR}=\frac{\alpha_{l}\left[a_{t} \neq 1\right]}{\alpha_{l}\left[a_{t}=1\right]}
$$

The second is the ratio between the transversal dispersivity (TDR) derived for an anisotropic case and the one obtained in the corresponding isotropic case:

$$
\operatorname{TDR}=\frac{\alpha_{t}\left[a_{t} \neq 1\right]}{\alpha_{t}\left[a_{t}=1\right]}
$$

\section{Results and Discussion}

\subsection{Anisotropic Conductivity Tensor}

Equations (2), (7), and (8) express the results of the upscaling procedures described in section 2.2. The macroscopic anisotropy factor $A_{1}$ is constant for all saturation degrees. Therefore anisotropy at the REV scale is completely defined by a saturated conductivity tensor. The macroscopic anisotropy factors $A_{2}$ and $A_{3}$ are functions of the capillary pressure head. The scaling procedure captures the tensorial nature of the relative conductivity function. In the following we analyze the relations $A_{2}(\Psi)$ and $A_{3}(\Psi)$ in more detail.

Figure 2a shows the pressure dependence of the anisotropy factors $A_{2}$, and Figure $2 \mathrm{~b}$ shows the pressure dependence of $A_{3}$ for microscopic anisotropy factors $a_{2,3}=0.2,0.5,1,2,5$, and 7.07. $A_{2}(0)=a_{2}^{2}$ and $A_{3}(0)=a_{3}$ represent the ratios between the two main components of the saturated conductivity tensor defined at the REV level for given $a_{2,3}$. The curves plotted in Figure 2 show an interesting feature: These anisotropic media are more conductive in one direction at highpressure values but become more conductive in the perpen- dicular direction at low-pressure values. The switching point between these two different regimes moves to lower-pressure values (to smaller water saturation) for increasing $a_{2}$ or $a_{3}$. When $a_{2}=a_{3}$, the switching point of the $A_{2}$ function takes place for lower pressures with respect to the switching point of the $A_{3}$ function. At the point where the anisotropy factor equals one, the medium shows isotropic behavior. The existence of a switching point separating the two complementary

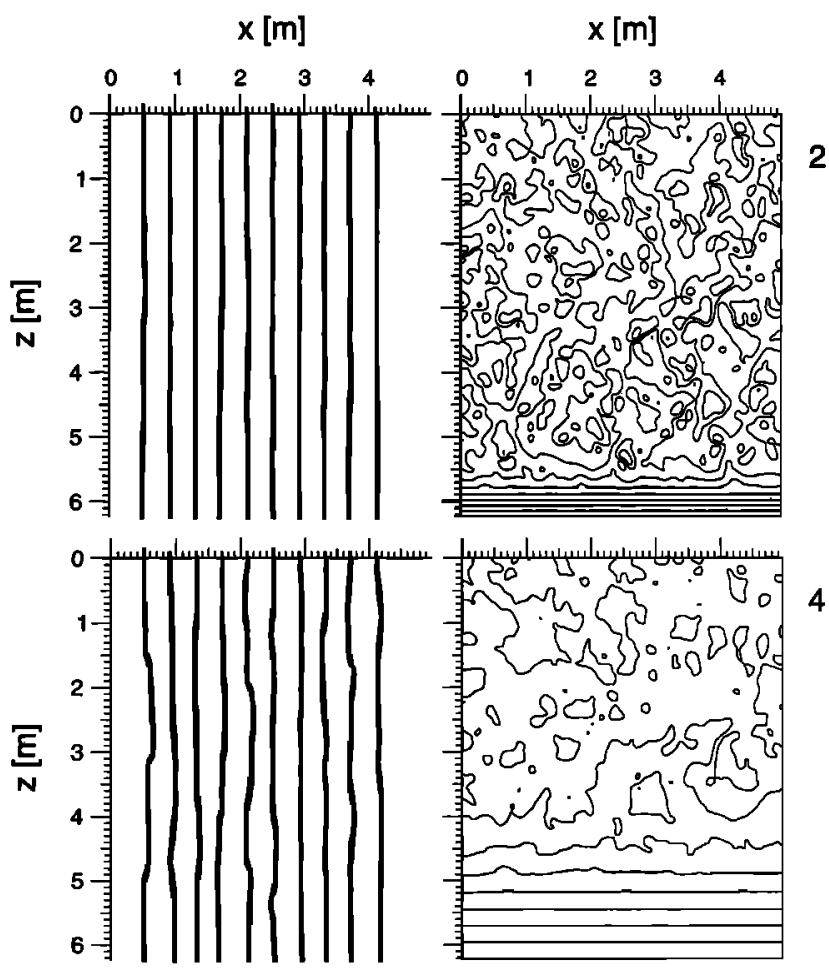

Figure 3. Trajectories and isobars. Macroscopic anisotropy factor is $A_{1}=10$ (saturation independent). Here $a_{1}=0.1$, with $-\log \left(j_{w}^{0} / K_{s z}\right)=2$ and 4 (these numbers are reported on the right side). 
regimes is related to the directionally dependent pore size distribution. For lower-pressure values, water moves mainly through the smaller pores, and the medium is more conductive in the direction of their higher abundance. At higher pressure (closer to water saturation) the larger pores are water-filled, and water might more easily flow in the other direction.

\subsection{Steady State Flow and Transport in Unsaturated Miller-Similar Media}

We will solve the transport problem for the media characterized by anisotropic average number of pores and by anisotropic pore size distribution only. For the first model of anisotropy, with a pressure-independent anisotropy factor $A_{1}$, two cases were considered: vertical grain orientation $\left(a_{1}=\right.$ 0.1 ), shown in Figure 3, and horizontal grain orientation $\left(a_{1}=10\right)$, shown in Figure 4. For each value of $a_{1}$ the transport problem was solved for two different upper boundary conditions, namely, $-\log \left(j_{w}^{0} / K_{s z}\right)=2$ and 4 , where $j_{w}^{0}$ is the imposed water flux at the surface. For the medium characterized by a pressure-dependent anisotropy factor $A_{2}$, four different water fluxes $j_{w}^{0}$ were considered: $-\log \left(j_{w}^{0} / K_{s z}\right)=1,2$, 3 , and 4. In the reported examples the microscopic anisotropy factor was $a_{2}=7.07$ (Figure 5). When the medium is saturated, the macroscopic anisotropy factor is $A_{2}=K_{s z} / K_{s x}=$ 50. The corresponding isotropic cases are shown in Figure 6.

Tables 1 and 2 list, for all the cases, the water fluxes, the corresponding average values of capillary pressure, which do not depend on anisotropy, and the average values of the tortuosity $\tau$ that were computed, for the generic particle, using the following expression:

$$
\tau=\left(\ell / \ell_{\min }\right)^{2}
$$
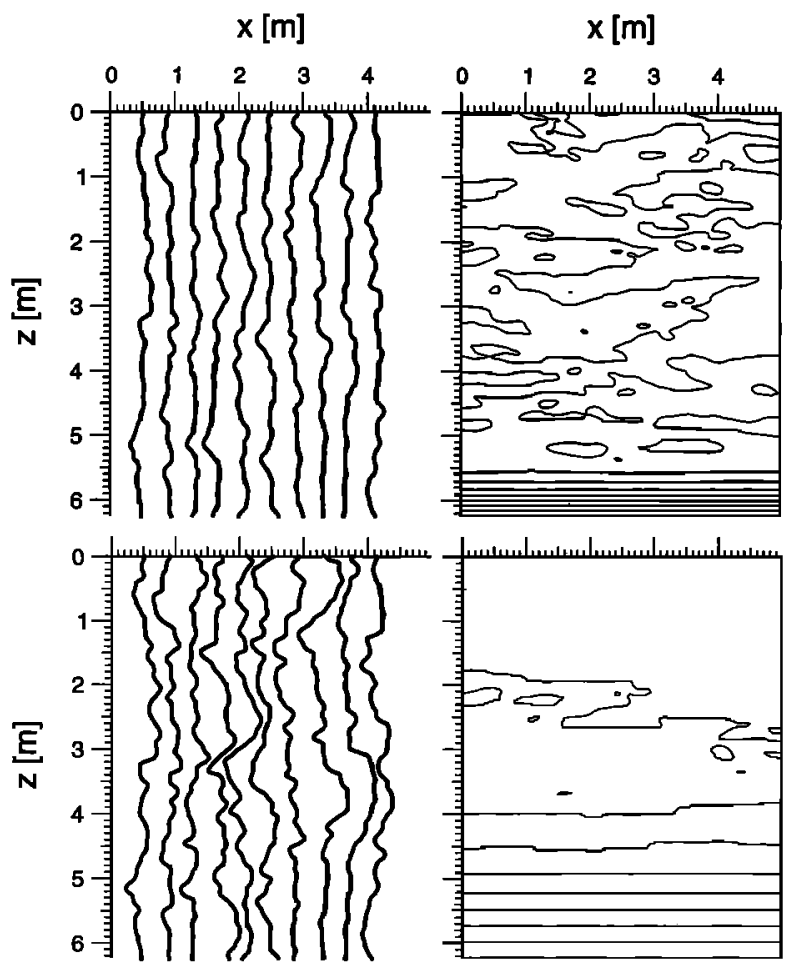

Figure 4. Trajectories and isobars. Macroscopic anisotropy factor is $A_{1}=0.1$ (saturation independent). Here $a_{1}=10$, with $-\log \left(j_{w}^{0} / K_{s z}\right)=2$ and 4 (these numbers are reported on the right side).
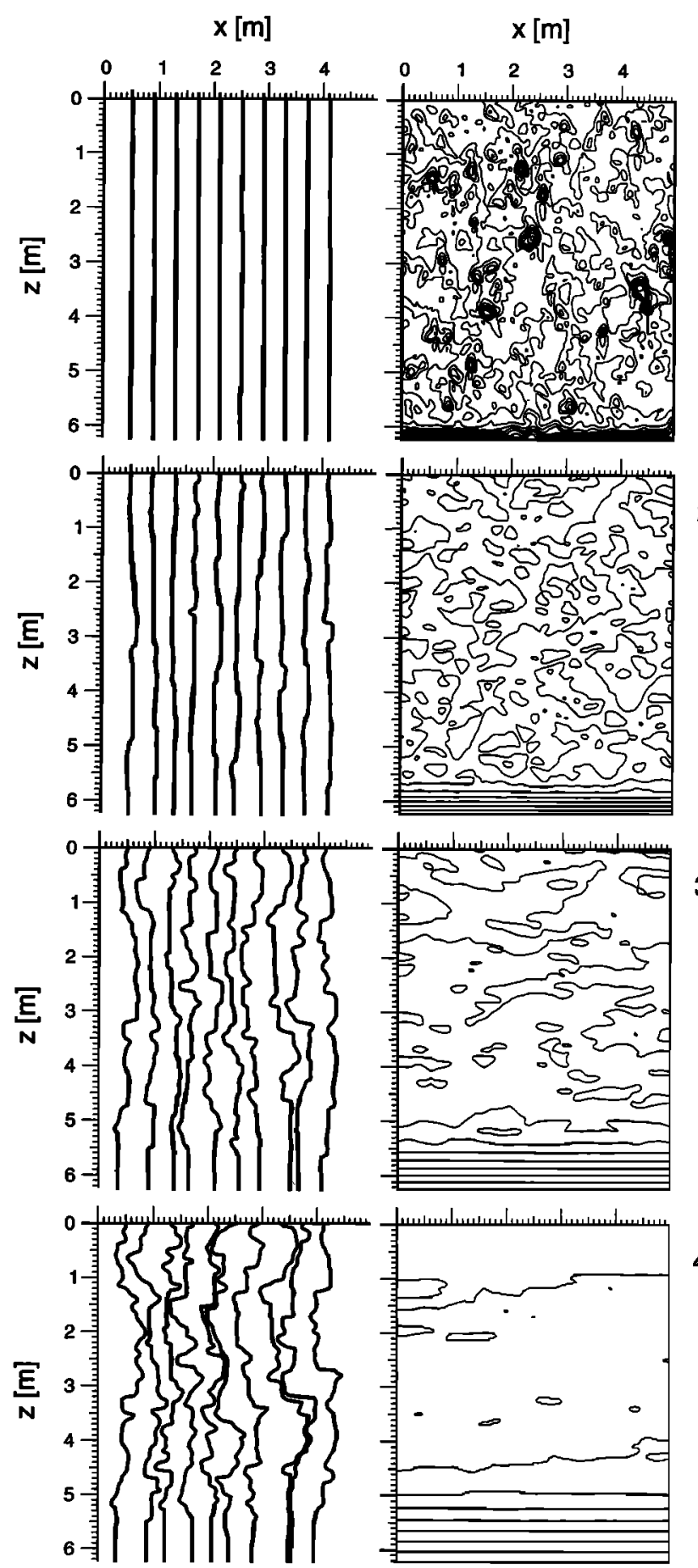

4 Figure 5. Trajectories and isobars. Macroscopic anisotropy factor $A_{2}$ is dependent on saturation. Here $a_{2}=7.07$, with $-\log \left(j_{w}^{0} / K_{s z}\right)=1,2,3$, and 4 (these numbers are reported on the right side).

where $\ell$ is the effective travel distance of the generic trajectory and $\ell_{\min }$ is the straight line between the starting and ending points of this trajectory.

Some of the main results derived for a medium that is macroscopically homogeneous and microscopically heterogeneous, isotropic, and Miller-similar [Roth, 1995; Roth and Hammel, 1996] hold as well for the analyzed anisotropic Miller-similar media. They are summarized in the following: (1) A 


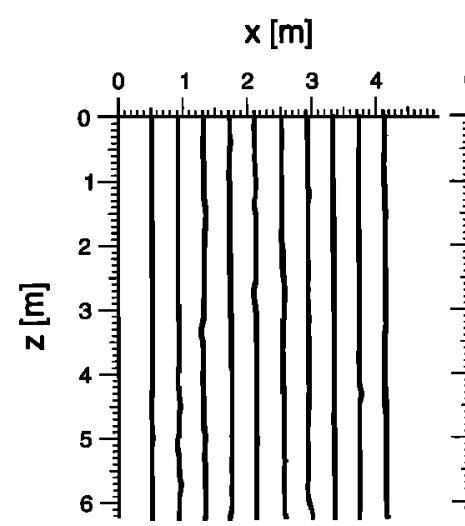

$$
x[\mathrm{~m}]
$$
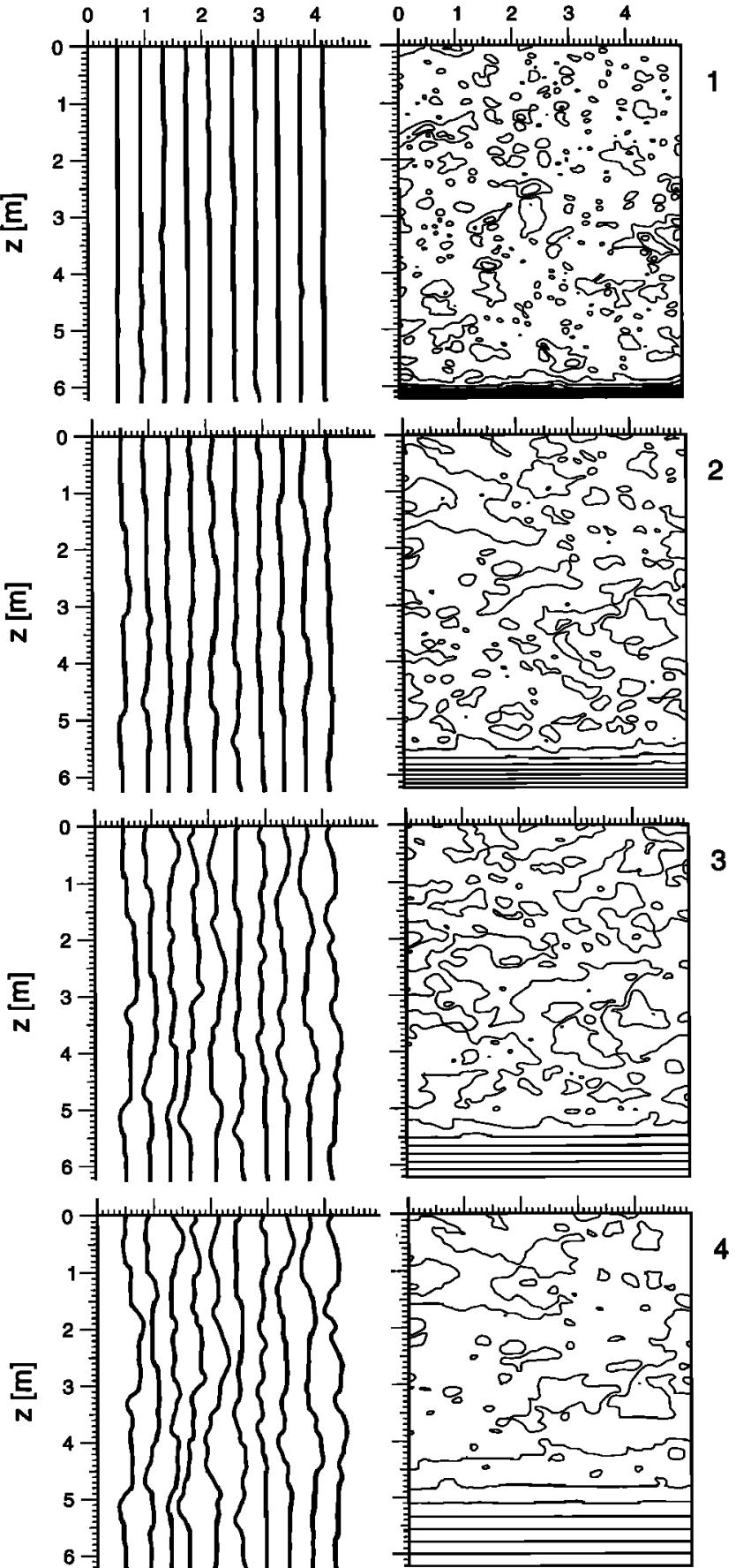

Figure 6. Trajectories and isobars. Isotropic case is shown with $-\log \left(j_{w}^{0} / K_{s z}\right)=1,2,3$, and 4 (these numbers are reported on the right side).

Table 1. Summary of Simulations for Constant Macroscopic Anisotropy Factor

\begin{tabular}{lcccc}
\hline & & \multicolumn{3}{c}{ Tortuosity } \\
\cline { 3 - 5 }$\left(j_{w}^{0} / K_{s z}\right)$ & Pressure, $\mathbf{m}$ & $a_{1}=1$ & $a_{1}=10$ & $a_{1}=0.1$ \\
\hline 2 & -0.5 & 1.1 & 1.2 & 1 \\
4 & -1.6 & 1.1 & 1.4 & 1 \\
\hline
\end{tabular}

Table 2. Summary of Simulations for Pressure-Dependent Macroscopic Anisotropy Factor

\begin{tabular}{cccc}
\hline & & \multicolumn{2}{c}{ Tortuosity } \\
\cline { 3 - 4 }$\left(j_{w}^{0} / K_{s z}\right)$ & Pressure, m & $a_{2}=1$ & $a_{2}=7.07$ \\
\hline 1 & -0.2 & 1 & 1 \\
2 & -0.5 & 1.1 & 1.1 \\
3 & -0.9 & 1.1 & 1.3 \\
4 & -1.6 & 1.1 & 1.6 \\
\hline
\end{tabular}

complex network of flow channels exists. (2) The topology of the network varies with saturation degree, and there are two complementary states separated by a critical point at which flow structures disappear (for the parameters chosen, the critical point appears approximatively for $-\log \left(j_{w}^{0} / K_{s z}\right)=1$ [Roth, 1995]). (3) The asymptotic state for transport is reached after traveling about 10 correlation lengths of the underlying structure. (4) The asymptotic longitudinal dispersivity depends on the water flux and has a minimum near the critical point.

For the rest of this paper we focus on features related to anisotropy. A first qualitative statement about the topography of trajectories and isobars is the following. A medium characterized by pore-scale anisotropy exhibits macroscopically a ten-

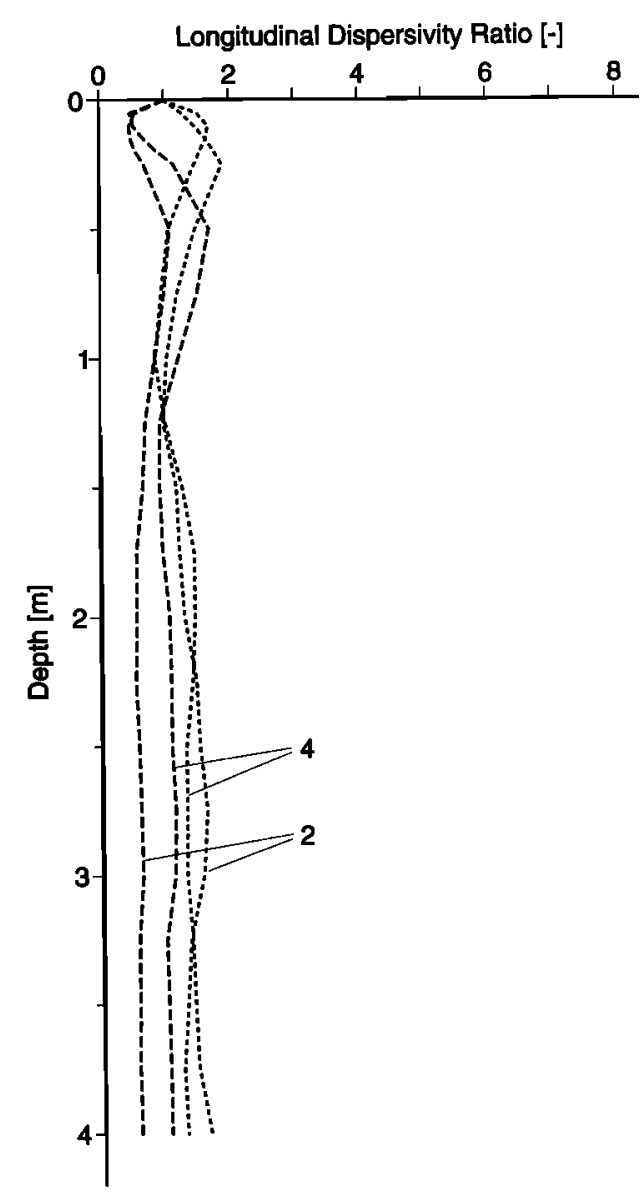

Figure 7. Ratio between the longitudinal dispersivity in an anisotropic medium with a constant anisotropy factor $A_{1}$ and the longitudinal dispersivity in an isotropic medium for two different water flow rates: $-\log \left(j_{w}^{0} / K_{s z}\right)=2$ and 4 (these numbers are reported in Figure 7). Long and short dashes correspond to the cases $a_{1}=10$ and $a_{1}=0.1$, respectively. 
dency to behave like a "column" system or a "layer system," depending on the direction of the higher conductivity being vertical or horizontal. The term column is inspired mainly by the geometry of the trajectories in a medium that is more conductive in the vertical direction, and the term layer is inspired by the pressure distribution aspect of a medium that is more conductive in the horizontal direction. When the macroscopic anisotropy factor remains constant for different flow rates, a particular soil behaves always either like a column system or like a layer system. When the anisotropy factor depends on the mean pressure, the same medium, for different water flows, switches from a column system at one flow rate to a layer system at another flow rate. In between, at the switching point, it behaves isotropically. In the following we will summarize the main properties of these two different regimes.

The capillary pressure head exhibits large-scale structures that differ considerably between the column and the layer system. In the column system the pressure tends to compensate the random distribution of the scaling factors in the vertical direction, leading to large pressure gradients. In the layer system the pressure tends to be constant in the horizontal direction. The trajectories are almost straight and vertical in the column regime and are more tortuous in the layer system as

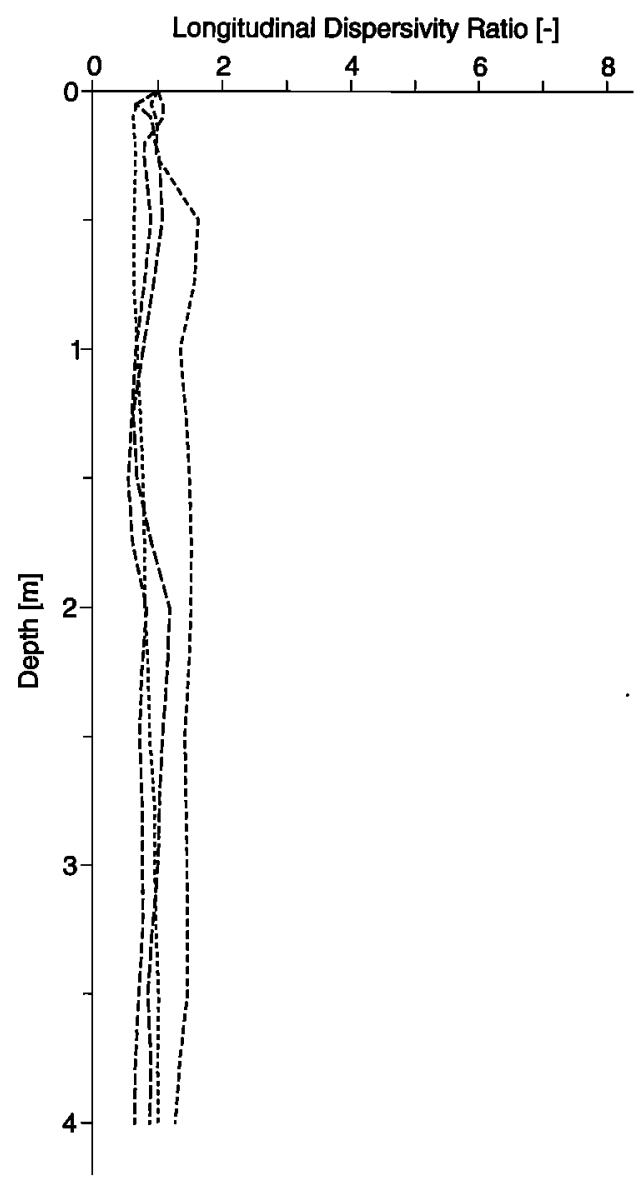

Figure 8. Ratio between the longitudinal dispersivity in an anisotropic medium with a pressure-dependent anisotropy factor $A_{2}$ and the longitudinal dispersivity in an isotropic medium for four different water flow rates: $-\log \left(j_{w}^{0} / K_{s z}\right)=1,2,3$, and 4. The microscopic anisotropy factor is $a_{2}=7.07$ leading to a macroscopic anisotropy factor $A_{2}=50$ at saturation. Increasing dash lengths correspond to decreasing water fluxes.

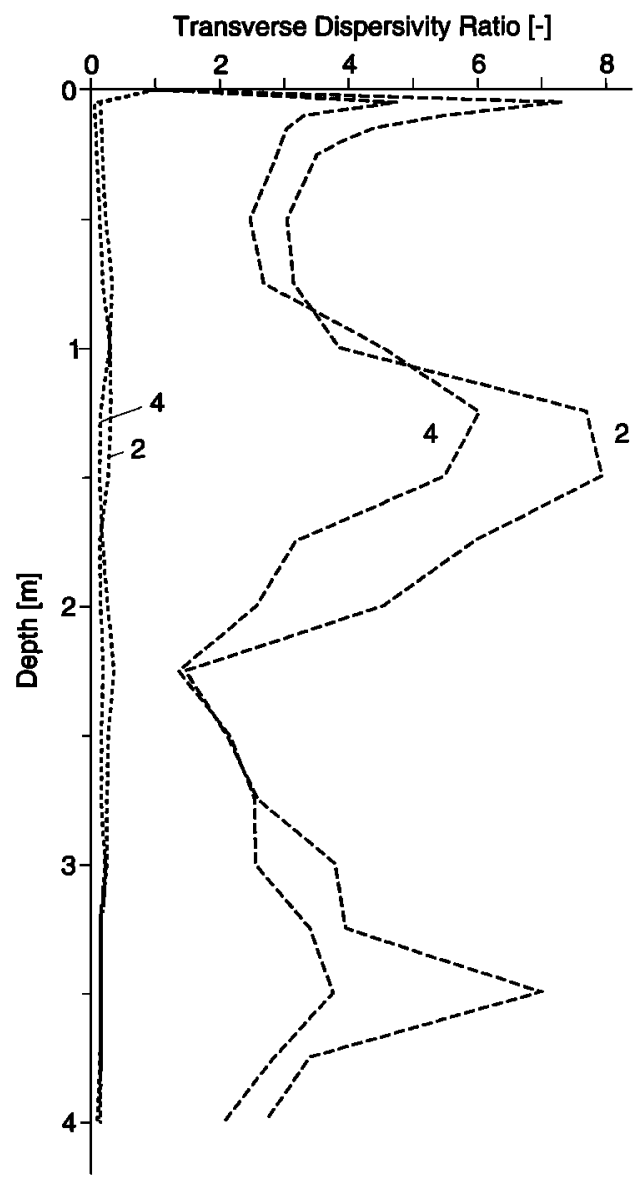

Figure 9. Ratio between the transverse dispersivity in an anisotropic medium with a constant anisotropy factor $A_{1}$ and the transverse dispersivity in an isotropic medium for two different water flow rates: $-\log \left(j_{w}^{0} / K_{s z}\right)=2$ and 4 (these numbers are reported in Figure 9). Long and short dashes correspond to the cases $a_{1}=10$ and $a_{1}=0.1$, respectively.

depicted in Figures 4-6. It is important to note that the switching point between the layer and the column system is not identical to the critical point between the "coarse-texture highconductivity" and the "fine-texture high-conductivity" regimes as defined by Roth [1995]. At the critical point the medium behaves almost homogeneously; at the switching point it behaves almost isotropically. Close to the critical point, the effect of anisotropy becomes negligible, since the network structure of the flow channels disappears, and, for the particular boundary conditions considered, the flow field tends to be onedimensional. Far from the critical point, the pore-scale anisotropy has a striking effect on the conductivity, on the topology of the trajectories, and on the transversal dispersivity compared to the corresponding isotropic case, as will be shown in section 3.3 .

\subsection{Dispersivity Ratios}

The longitudinal and transversal dispersivity values (equations (12) and (13)) were used to estimate a second index of anisotropy at the field scale: the ratio between the dispersivity evaluated for the anisotropic case and that evaluated for the corresponding isotropic case (assuming $a_{1}=1$ ) as stated in (14) and (15). The values of these dispersivity ratios are plotted as a function of depth. Figures 7 and 8 depict the longitudinal 


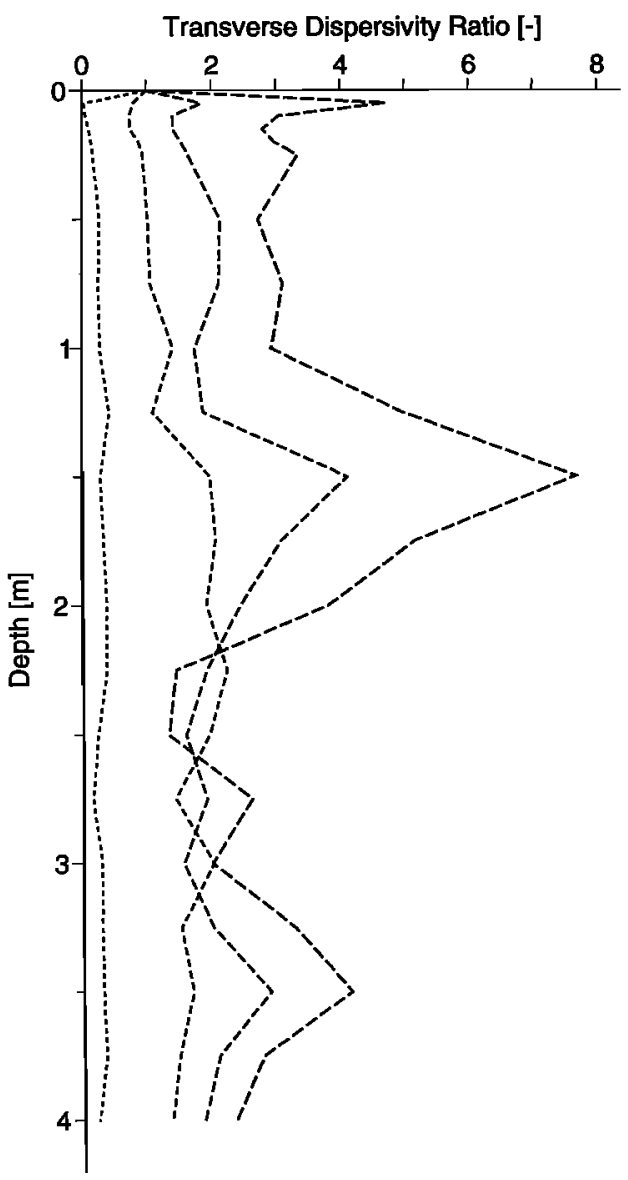

Figure 10. Ratio between the transverse dispersivity in an anisotropic medium with a pressure-dependent anisotropy factor $A_{2}$ and the transverse dispersivity in an isotropic medium for four different water flow rates: $-\log \left(j_{w}^{0} / K_{s z}\right)=1,2,3$, and 4. The microscopic anisotropy factor is $a_{2}=7.07$ leading to a macroscopic anisotropy factor $A_{2}=50$ at saturation. Increasing dash lengths correspond to decreasing water fluxes.

dispersivity ratio values for the constant $\left(A_{1}\right)$ and for the pressure-dependent $\left(A_{2}\right)$ macroscopic anisotropy factors, respectively. Transversal dispersivity ratios are plotted for the same cases in Figures 9 and 10 . Note that the underlying structure for the various flow field simulations always corresponded to the same realization.

In the examined cases the longitudinal dispersivity does not seem to be influenced by variations of the microscopic anisotropy factors at different flow rates (Figures 7 and 8 ). In other words, the variability of travel times at a given flow rate seems to be independent of the lateral displacement due to anisotropy. A systematic variation of the transversal dispersivity is induced by anisotropy (Figures 9 and 10). The dispersivity ratios clearly depend on the value of the macroscopic anisotropy factor $A_{1}$ or $A_{2}$. When the macroscopic anisotropy factor is pressure-dependent $\left(A_{2}\right)$, the dispersivity ratio, for decreasing water flux, shifts from values less than 1 to values larger than 1 . In the case $A_{1}=10$ it is evident that lower water flux leads to higher tortuosity (Table 1 ) and higher transverse dispersivity ratio (Figure 9). When $A_{1}=0.1$, because of anisotropy, trajectories are straight vertical ( $\tau=1$ in Table 1$)$, and the transverse dispersivity ratio does not depend on the water flux (Figure 9). Water flows in a complex network of channels which have a characteristic length [Roth, 1995]. The periodicity of the transverse dispersivity ratio and the tortuosity of the trajectories can be related to this "channel length." The significant fluctuations of the ratio between transversal dispersivities with depth can be attributed to nonergodicity. For our particular realization the mean trajectory (not shown) is not vertical, when not forced by anisotropy. Deviation depends on anisotropy. In ergodic conditions the transversal dispersivity is proportional to the spreading around the trajectory of the ensemble centroid [Dagan, 1989]. In our case, as a consequence of nonergodicity, dispersivity is partly overestimated with respect to the ergodic case because the center of mass of the plume does not travel vertically along the mean direction of flow. To test this assertion, we computed the variance of displacement around the nonzero mean transversal displacement instead of around the injection point, deriving an "effective transversal dispersivity ratio." This effective transversal dispersivity ratio shows less pronounced fluctuations (Figures 11 and 12).

\section{Conclusions}

In this paper we demonstrated the striking effects of microscale anisotropy on hydraulic conductivity and transverse

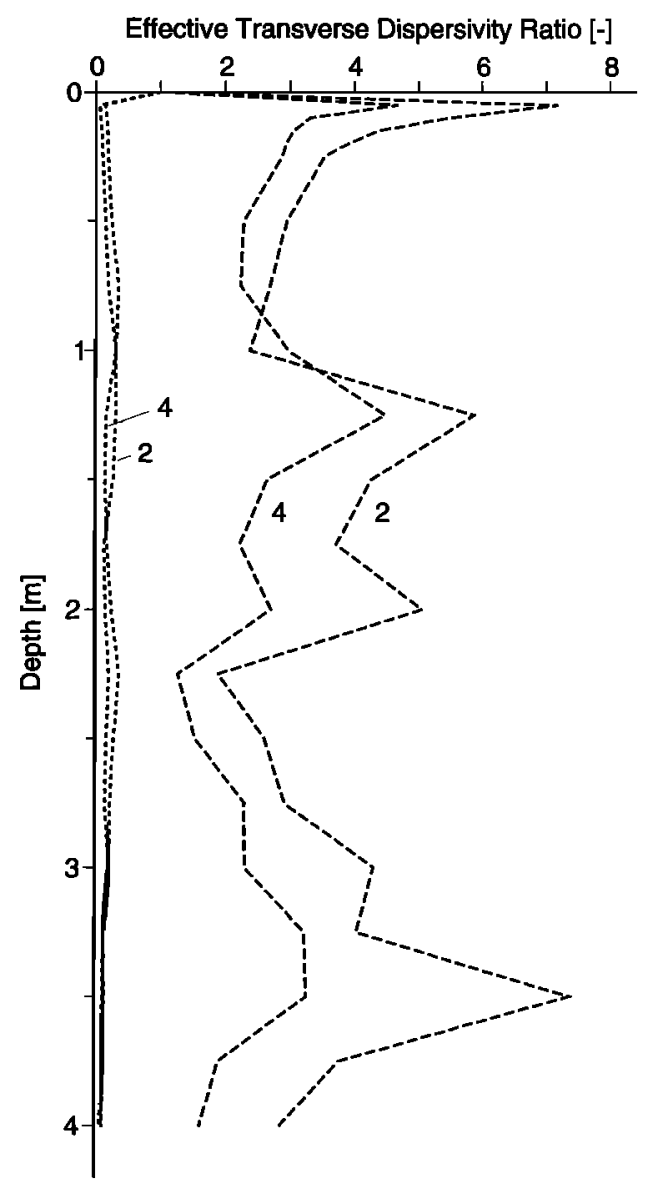

Figure 11. Ratio between the variance of the displacement around the mean transverse displacement in an anisotropic medium with a constant anisotropy factor $A_{1}$ and the variance of the displacement around the mean transverse displacement in an isotropic medium for two different water flow rates: $-\log$ $\left(j_{w}^{0} / K_{s z}\right)=2$ and 4 (these numbers are reported in Figure 11). Long and short dashes correspond to the cases $a_{1}=10$ and $a_{1}=0.1$, respectively. 


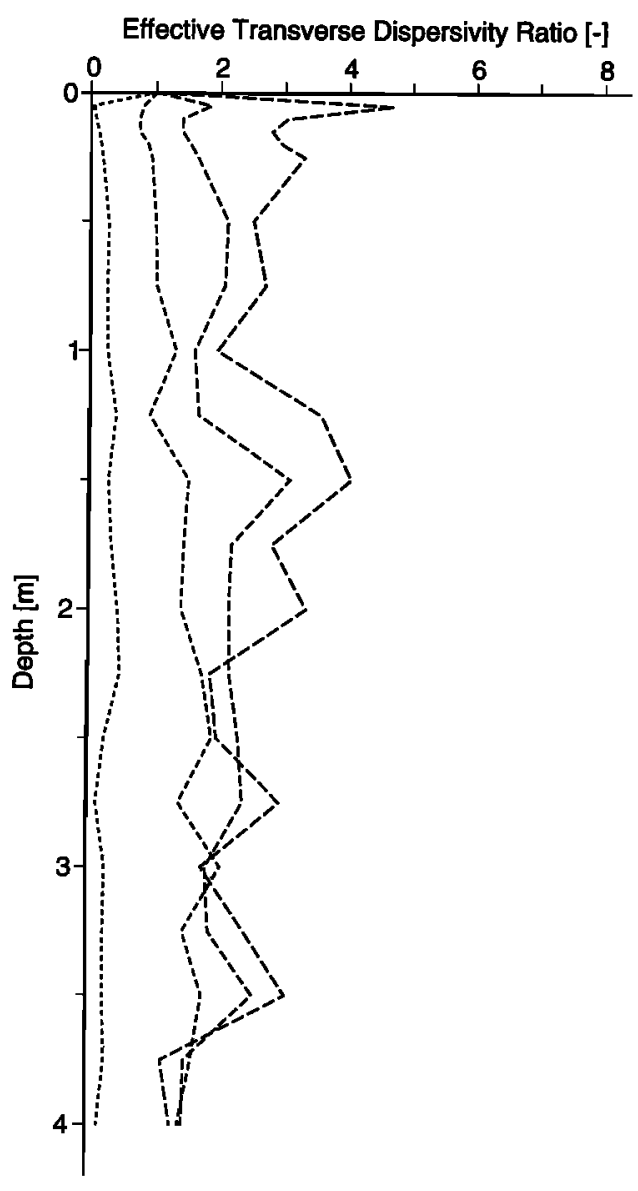

Figure 12. Ratio between the variance of the displacement around the mean transverse displacement in an anisotropic medium with a pressure-dependent anisotropy factor $A_{2}$ and the variance of the displacement around the mean transverse displacement in an isotropic medium for four different water flow rates: $-\log \left(j_{w}^{0} / K_{s z}\right)=1,2,3$, and 4 . The microscopic anisotropy factor is $a_{2}=7.07$ leading to a macroscopic anisotropy factor $A_{2}=50$ at saturation. Increasing dash lengths correspond to decreasing water fluxes.

dispersivity in a very simple case: that of steady state flow, no subscale dispersion, and main flow parallel to the structure bedding. From lateral dispersion observed in field experiments, anisotropic conductivities can be inferred, but detection of the subscale (pore scale) properties of the soil, that could provide an explanation for the macroscopic anisotropic behavior, is in many cases experimentally not possible. Therefore models that link microscopic soil properties with macroscopically observable parameters like dispersivity can help to accept or reject structural hypotheses.

We compared conceptual models of anisotropy at the pore scale applying Miller similarity. One of the pore-scale models leads to a constant anisotropy factor expressed as the ratio between conductivities in the two main directions. The other models yield anisotropy factors which strongly depend on saturation. In particular, the conductivity ratios switch from values below 1 to values above 1 or vice versa, depending on saturation. In the examined cases only the transverse dispersivities were considerably affected by anisotropy, whereas the longitudinal dispersivities remained more or less invariant. Note, however, that relatively large variations of the conduc- tivity ratios lead only to relatively small variations of the transverse dispersivity ratios as compared to the isotropic cases, when local dispersion is neglected.

We would like to stress the fact that the correlation structure in this analysis was isotropic. It is well known that a macroscopic anisotropic pressure-dependent behavior may also originate from the presence of thin layers of different materials [Mualem, 1985], or, more generally, from an anisotropic correlation structure [Desbarats, 1998; Yeh et al., 1985a, b]. Here we demonstrate the effect of pore geometry of an idealized, inert, and nondeformable Miller-similar microstructure on flow and transport. The geometry of the microstructure can lead to anisotropic behavior at the larger scale, even if the system is characterized by an isotropic correlation structure. Anisotropic correlation structure of hydraulic parameters and anisotropy in the pore structure do not lead to the same macroscopic behavior. Both types of anisotropy can be modeled by assuming a properly upscaled anisotropic, saturationdependent conductivity tensor, but the dependence of the conductivity tensor on the water content is different for the two concepts of anisotropy. An anisotropic pore size distribution gives a conductivity ratio that is larger or lower than 1 , depending on saturation; the medium acts as a column as well as a layer system. Such a switching behavior cannot be reproduced by assuming anisotropy in the correlation structure: the REV turns out to be always more conductive in the direction parallel to the bedding [Mualem, 1985; Yeh et al., 1985b; Russo, 1995a, b; Desbarats, 1998]. For this reason, comparing experimental observations with simulation results will allow discrimination between a model based on anisotropic correlation structures and a conceptual model based on anisotropy of the void space of the medium. The results obtained suggest in addition the importance of investigating microstructures to justify or reject the assumptions about the pore space. This will avoid that such models be used as a black box. Finally, the pore-scale models may also help to design artificial porous media with well-defined anisotropic characteristics.

\section{References}

Bear, J., Dynamics of Fluids in Porous Media, Dover, Mineola, N. Y., 1972.

Bear, J., C. Braester, and P. C. Menier, Effective and relative permeabilities of anisotropic porous media, Trans. Porous Media, 2, 301316, 1987.

Dagan, G., Flow and Transport in Porous Formations, Springer-Verlag, New York, 1989.

Desbarats, A. J., Scaling of constitutive relationships in unsaturated heterogeneous media: A numerical investigation, Water Resour. Res., 34(6), 1427-1435, 1998.

Friedman, S. P., and N. A. Seaton, On the transport properties of anisotropic networks of capillaries, Water Resour. Res., 32(2), 339$347,1996$.

Green, T. R., and D. L. Freyberg, State-dependent anisotropy: Comparison of quasi-analytical solutions with stochastic results for steady gravity drainage, Water Resour. Res., 31(9), 2201-2211, 1995.

Hubbert, M. K., Darcy's law and the field equations of the flow of underground fluids, Trans. Am. Inst. Min. Metall. Pet. Eng., 207, 222-239, 1956.

McCord, J. T., D. B. Stephens, and J. L. Wilson, Toward validating state-dependent macroscopic anisotropy in unsaturated, media: Field experiments and modeling considerations, J. Contam. Hydrol., 7, 145-175, 1990.

Miller, E. E., and R. D. Miller, Physical theory for capillary flow phenomena, J. Appl. Phys., 27, 324-332, 1956.

Mualem, Y., Anisotropy of unsaturated soils, Soil Sci. Soc. Am. J., 48, 505-509, 1985.

Roth, K., Steady state flow in an unsaturated, two-dimensional, mac- 
roscopically homogeneous Miller-similar medium, Water Resour. Res., 31(9), 2127-2140, 1995.

Roth, K, and K. Hammel, Transpport of conservative chemical through an unsaturated two-dimensional Miller-similar medium with steady state flow, Water Resour. Res., 32(6), 1653-1663, 1996.

Russo, D., Stochastic analysis of simulated vadose zone solute transport in a vertical cross section of a heterogeneous soil during nonsteady infiltration, Water Resour. Res., 27(3), 267-283, 1991.

Russo, D., On the velocity covariance and transport modeling in heterogeneous anisotropic porous formations, 1, Saturated flow, Water Resour. Res., 31(1), 129-137, 1995 a.

Russo, D., On the velocity covariance and transport modeling in heterogeneous anisotropic porous formations, 2, Unsaturated flow, $\mathbf{W a}$ ter Resour. Res., 31(1), 139-145, 1995b.

Sposito, G., and W. A. Jury, Miller similitude and generalized scaling analysis, in Scaling in Soil Physics: Principles and Applications, SSSA Spec. Publ. 25, 1990.

Stephens, D. B., and S. Heermann, Dependence of anisotropy on saturation in a stratified sand, Water Resour. Res., 24(5), 770-778, 1988.

Tseng, T.-H., and W. A. Jury, Comparison of transfer function and deterministic modeling of area-averaged solute transport in a heterogeneous field, Water Resour. Res., 30(7), 2051-2063, 1994. van Genuchten, M. T., A closed-form equation for predicting the hydraulic conductivity of unsaturated soils, Soil Sci. Soc. Am. J., 44, 892-898, 1980.

Yeh, T.-C., L. W. Gelhar, and A. L. Gutjahr, Stochastic analysis of unsaturated flow in heterogeneous soils, 1 , Statistically isotropic media, Water Resour. Res., 21(4), 447-456, 1985a.

Yeh, T.-C., L. W. Gelhar, and A. L. Gutjahr, Stochastic analysis of unsaturated flow in heterogeneous soils, 2 , Statistically anisotropic media with variable $\alpha$, Water Resour. Res., 21(4), 457-464, $1985 \mathrm{~b}$.

H. Flühler, T. Gimmi, and N. Ursino, Soil Physics, Swiss Federal Institute of Technology Zurich, Grabenstrasse 3/11a, Schlieren CH8952, Switzerland. (fluehler@ito.umnw.ethz.ch; gimmi@ito.umnw. ethz.ch; ursino@ito.umnw.ethz.ch)

K. Roth, Institute of Environmental Physics, University of Heidelberg, Heidelberg D-69120, Germany. (kurt.roth@urz.uni-heidelberg. de)

(Received August 30, 1999; revised October 6, 1999; accepted October 27, 1999.) 